\title{
Levofloxacin Induced Proximal Myopathy
}

\section{Bathish Jlal ${ }^{1}$, Buzabarov Galina ${ }^{2}$ and Zeidman Aliza ${ }^{1,2}$}

${ }^{1}$ Department of Medicine B, Hasharon Hospital, Rabin Medical Center, Israel

${ }^{2}$ Petah-Tikva, Sackler Medical School, Tel-Aviv University, Israel

\section{Introduction}

Levofloxacin is a synthetic antibiotic of the Fluroquinolones group, used to treat severe bacterial infections that have failed to respond to other antibiotics. Levofloxacin is associated with serious and life threatening adverse reactions as well as spontaneous tendon ruptures and irreversible peripheral neuropathy. We present a patient who developed proximal myopathy during levofloxacin treatment, which resolved shortly after the treatment was discontinued.

\section{Case Report}

A healthy 46 year old male presented with fever up to 390 Celsius and general weakness for the last two weeks. Physical examination at presentation was normal. Neurological examination revealed no neurological deficits. Laboratory tests showed elevated erythrocyte sedimentation rate $-90 \mathrm{~mm} \mathrm{Hg}$, leukocytosis 14,000 (90\% neutrophils), liver, renal function test and chest $\mathrm{x}$-ray were normal. The patient was diagnosed as fever of unknown origin (FUO). Extensive evaluation including blood and urine cultures, viral serology and Echocardiogram was conducted. Because of the fever, general weakness and leukocytosis, we started treatment with Ceftriaxone 1 gr. After 3 days of no improvement the treatment was replaced by levofloxacin 500 $\mathrm{mg} / \mathrm{d}$ for 7 days. The patient's condition has improved and he was discharged with instructions to complete 2 more days of treatment with levofloxacin at home.

A day after his discharge, the patient was readmitted with complains of severe weakness and inability to walk. He underwent additional diagnostic tests including neurological examination which suspected proximal neuropathy. Blood tests for electrolytes, viral and collagen serology, computerized tomography (CT) of brain and lumbar puncture, were all normal. In addition he had electromyography (EMG) test which demonstrated myopathy changes of the proximal legs muscles.
Treatment with levofloxacin was stopped and a dramatic improvement was noticed within a few days. The patient regained the ability to walk and there were no further complains on a monthly follow up.

\section{Discussion}

Due to extensive developments in the synthesis and clinical use of Fluroquinolones, it is now considered broad spectrum antimicrobial activity with pharmacokinetics features and toxicity profiles.

The most common adverse reactions of levofloxacin include gastrointestinal and central nervous system symptoms such as nausea, abdominal discomfort, headache, insomnia and fatigue [1].

In the last decade several case reports of myopathies [2] including tendinitis and tendon rupture after the use of flouroquinolones, has been published $[3,4]$. To our knowledge this is the first case report in Israel on severe reversible proximal myopathy related to the use of levofloxacin. The mechanism is not clear and required additional investigation. However, Physicians should be aware of this severe adverse reaction.

\section{References}

1. Lipsky BA, Baker CA (1999) Fluoroquinolone toxicity profiles: a review focusing on newer agents. Clin Infect Dis 28: 352-364.

2. Mor A, Mitnick HJ, Pillinger MH, Wortmann RL (2009) Drug-induced myopathies Bull NYU Hosp Jt Dis 67: 358-369.

3. Durey A, Baek YS, Park JS, Lee K, Ryu JS, et al. (2010) Levofloxacin-induced Achilles tendinitis in a young adult in the absence of predisposing conditions. Yonsei Med J 51: 454-456.

4. Fleisch F, Hartmann K, Kuhn M (2000) Fluoroquinolone-induced tendinopathy: also occurring with levofloxacin. Infection 28: 256-257. 